\title{
Cyclization Reactions of Mono-Thiocarbohydrazones with $\alpha$ - Haloketones: Synthesis and Potential Biological Activities of Substituted 1,3-thiazoles and 1,3,4-thiadiazines
}

\author{
Ababneh and Ahmad Maslat \\ ${ }^{a}$ Chemistry Department, Yarmouk University, Irbid - Jordan. \\ ${ }^{b}$ Chemistry Department, Qatar University, Qatar. \\ ${ }^{c}$ Biology Department, Yarmouk University, Irbid-Jordan.
}

Hasan I. Tashtoush ${ }^{\mathrm{a}^{*}}$, Mahmoud Al-Talib ${ }^{\mathrm{a}}$, Mohanad Shkoor ${ }^{\mathrm{b}}$, Bushra

Received: $6^{\text {Th }}$ May 2021; Accepted: $29^{\text {th }}$ July 2021

\begin{abstract}
A series of new substituted 1,3-thiazole derivatives 10a-h were prepared via the reaction of monothiocarbohydrazones 8a-h with one equivalent of $\alpha$-bromoketones in ethanol. Similarly, another series of new substituted 1,3,4-thiadiazines 11a-e were prepared from the reaction of monothiocarbohydrazones $\mathbf{8 i}, \mathbf{j}$ with one equivalent of $\alpha$-bromoketones in ethanol. The resulting products were obtained as colored crystals in moderate to good yields. All new compounds are fully characterized by ${ }^{1} \mathrm{H}-,{ }^{13} \mathrm{C}-\mathrm{NMR}$, IR and elemental analysis. Pharmacophore modeling study of four of the synthesized compounds revealed that at least two of them have potential biological activities. Some of these activities will be investigated experimentally in the lab.
\end{abstract}

Keywords: Thiocarbohydrazide, Thiocarbohydrazones, $\alpha$-haloketones, 1,3-thiazoles,1,3,4thiadiazines.

\section{Introduction}

Thiocarbohydrazide 1 (Figure 1) can be easily synthesized via the hydrazinolysis of carbon disulfide ${ }^{[1]}$. Compound $\mathbf{1}$ itself exerts a wide spectrum of pharmacological activities ${ }^{[2]}$. In addition, it is known to be a versatile organosulfur building block and has been widely used for the synthesis of organic heterocyclic ring systems $^{[2,3]}$, including substituted 1,2,4triazoles $^{[4-7]}$, triazines ${ }^{[8]}$, triazinones ${ }^{[9]}$ and thiadiazoles ${ }^{[10-12]}$.

It is well established that the acid-catalyzed condensation of $\mathbf{1}$ with one equivalent of carbonyl compounds $\mathbf{2}$ gives monothiocarbohydrazones, which exist in two tautomers, $\mathbf{3}$ or/and the cyclic form $\mathbf{4}$, depending on the starting carbonyl compound ${ }^{[13]}$. Recently, we demonstrated that subsequent cyclization of $\mathbf{3}$ or $\mathbf{4}$ with dialkyl acetylene dicarboxylate $\mathbf{5}$ gave the substituted 4-thiazolidinones $\mathbf{6}$ or 7, respectively $^{[14]}$, Scheme 1.

Organo-sulfur compounds are well established to exhibit a wide range of biological and pharmacological activities, such as antifunga ${ }^{[15]}$, antibacterial ${ }^{[16-18]}$ and antiinflammatory activities ${ }^{[19]}$. In specific, thiazolidinone moiety seems to be an interesting example of such sulfur-containing heterocyclic systems due to its wide range of biological activities. Compounds containing this moiety have been demonstrated to possess analgesic ${ }^{[20]}$, anti-tubercular ${ }^{[21]}$, antimicrobial and antifungal $^{[22]}$, antiviral ${ }^{[23]}$, anticancer and antioxidant $^{[24,25]}$, anticonvulsant ${ }^{[26]}$, anti-diabetic ${ }^{[26,27]}$ and antibacterial activities ${ }^{[28]}$. 


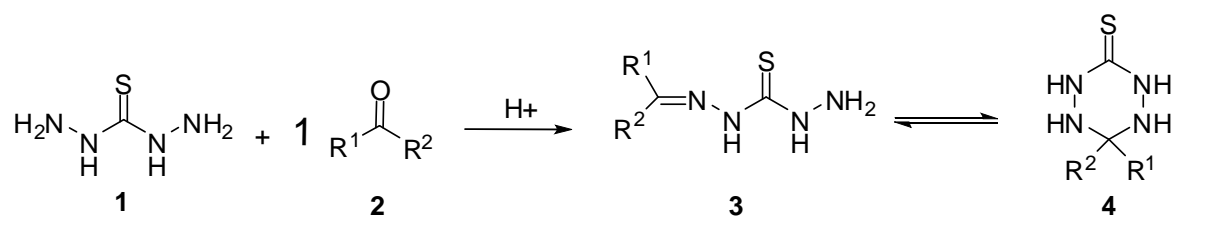

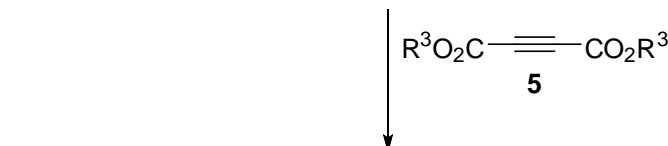

$$
\begin{aligned}
& \text { (n) }
\end{aligned}
$$

Scheme 1. Preparation of substituted 4-thiazolidinones (6 and 7) from thiocarbohydrazide (1).

Little is known about the reactions of mono-thiocarbohydrazones with $\alpha$-halo-carbonyl compounds $^{[29]}$. These facts encourage us to synthesize a series of new substituted thiazolidine derivatives via chemo-selective [3+2] cyclization reactions of mono-thiocarbohydrazones with $\alpha$-bromoketones. In the present report, we summarized our results on the synthesis and structural characterization of some thiazole and thiadiazine derivatives, as well as their potential biological activities using pharmacophore modeling approach.

\section{Materials and Methods}

Thiocarbohydrazide $\mathbf{1}$ and mono-thiocarbohydrazones 8a-j were prepared following literature procedures ${ }^{[31]}$. Melting points $\left({ }^{\circ} \mathrm{C}\right)$ were performed on open capillaries using an Electro thermal digital melting point apparatus and were uncorrected. Proton nuclear magnetic resonance $\left({ }^{1} \mathrm{H}-\mathrm{NMR}\right)$ spectra were collected using a Bruker Avance $400 \mathrm{MHz}$ spectrometer in deuterated chloroform $\left(\mathrm{CDCl}_{3}\right)$ or dimethyl sulfoxide $\left(\right.$ DMSO- $\left._{6}\right)$ with tetramethylsilane (TMS) as an internal standard. Carbon-13 nuclear magnetic resonance $\left({ }^{13} \mathrm{C}-\mathrm{NMR}\right)$ spectra were recorded on a Brucker Avance $75 \mathrm{MHz}$ spectrometer. Infrared spectra (IR) were recorded on Perkin Elmer FT-IR SP-2000 spectrometer as potassium bromide $(\mathrm{KBr})$ discs. All chemicals used in this work were purchased from Aldrich Chemicals and were used without further purification. Elemental analyses were carried out at Al AlBayt University laboratories, Jordan.
General procedure for the preparation of the 2-Aryledine hydrazino-4-arylthiazol-3-(2H)amines 10(a-h)

The mono-thiocarbohydrazone 8 (4 mmol) was added to a solution of phenacyl bromide (4 mmol) or 4-bromo phenacyl bromide (4 mmol) in ethanol $(20 \mathrm{ml})$. The reaction mixture was heated under reflux, for one hour. The reaction mixture was cooled to room temperature and the precipitated product was collected by filtration, washed with ethanol and ether and finally dried.

\section{2-(2-benzylidenehydrazono)-4-phenylthiazol- $3(2 H)$-amine (10a)}

Light yellow. Yield: 83\%, mp 194-195 ${ }^{\circ} \mathrm{C}$. $\operatorname{IR}\left(\mathrm{cm}^{-1}\right)$ : 1591-1607 ( $\mathrm{C}=\mathrm{N}$, aromatic), 3355$3482\left(\mathrm{NH}_{2}\right)$. ${ }^{1} \mathrm{H}-\mathrm{NMR}\left(400 \mathrm{MHz}, \mathrm{CDCl}_{3}\right): 7.21$ (1H, s, SCH), 7.51-7.56 (6H, m, CH), 7.72-7.79 (4H, m, CH), 8.63 (1H, s, NCH). ${ }^{13} \mathrm{C}-\mathrm{NMR}(75$ $\left.\mathrm{MHz}, \mathrm{CDCl}_{3}\right)$ : 104.9, 127.5, 127.8, 128.6, 129.1, 129.4, 129.9, 131.2, 132.8, 142.7, 151.0, 168.3. DEPT 135: 104.9, 127.5, 128.6, 129.1, 129.4, 129.9, 131.2, 151.0. Anal. Cacld. For $\left(\mathrm{C}_{16} \mathrm{H}_{14} \mathrm{~N}_{4} \mathrm{~S}, \%\right)$ : C, 65.31; H ,4.76; N, 19.05; S, 10.88. Found: C, 64.78; H, 5.06; N, 18.63; S : 10.66 .

\section{2-(2-benzylidenehydrazono)-4-p-tolylthiazol- 3(2H)-amine (10b)}

Light yellow. Yield: $70 \%, \mathrm{mp} 205-206{ }^{\circ} \mathrm{C}$. $\operatorname{IR}\left(\mathrm{cm}^{-1}\right)$ : 1563-1611 ( $\mathrm{C}=\mathrm{N}$, aromatic), 3240$3320\left(\mathrm{NH}_{2}\right) .{ }^{1} \mathrm{H}-\mathrm{NMR}\left(400 \mathrm{MHz}, \mathrm{CDCl}_{3}\right): 2.37$ $\left(3 \mathrm{H}, \mathrm{s}, \mathrm{CH}_{3}\right), 7.13(1 \mathrm{H}, \mathrm{s}, \mathrm{SCH}), 7.32-7.34(2 \mathrm{H}$, $\mathrm{d}, J=8 \mathrm{~Hz}, \mathrm{CH}), 7.54-7.56(3 \mathrm{H}, \mathrm{m}, \mathrm{CH}), 7.66-$ $7.68(2 \mathrm{H}, \mathrm{d}, J=8.1 \mathrm{~Hz}, \mathrm{CH}), 7.71-7.73(2 \mathrm{H}, \mathrm{m}$, $\mathrm{CH}), 8.55$ (1H, s, NCH). ${ }^{13} \mathrm{C}-\mathrm{NMR}(75 \mathrm{MHz}$, $\left.\mathrm{CDCl}_{3}\right)$ : 21.1, 104.3, 127.5, 127.9, 128.6, 129.3, 
129.7, 129.9, 130.2, 141.3, 142.7, 151.1, 168.3. DEPT 135: 21.1，104.3，127.5, 128.6, 129.3, 129.7, 129.9, 151.1. Anal. Cacld. For $\left(\mathrm{C}_{17} \mathrm{H}_{16} \mathrm{~N}_{4}\right.$ S. \%): Calc. (\%): C, 66.23; H, 5.19; N, 18.18; S, 10.39. Found: C, 66.28; H, 5.53; N, 17.96; S, 10.11 .

2-(2-benzylidenehydrazono)-4-(4-methoxyphenyl)thiazol-3(2H)-amine (10c)

Light yellow. Yield: $94 \%, \mathrm{mp} 188-189{ }^{\circ} \mathrm{C}$. $\operatorname{IR}\left(\mathrm{cm}^{-1}\right): 1580-1607(\mathrm{C}=\mathrm{N}$, aromatic), 3300$3400\left(\mathrm{NH}_{2}\right) .{ }^{1} \mathrm{H}-\mathrm{NMR}\left(400 \mathrm{MHz}, \mathrm{CDCl}_{3}\right): 3.87$ $\left(3 \mathrm{H}, \mathrm{s}, \mathrm{OCH}_{3}\right), 7.10-7.12(2 \mathrm{H}, \mathrm{d}, J=8.8 \mathrm{~Hz}, \mathrm{CH})$, $7.23(1 \mathrm{H}, \mathrm{s}, \mathrm{SCH}), 7.58-7.59(3 \mathrm{H}, \mathrm{m}, \mathrm{CH}), 7.75-$ $7.78(4 \mathrm{H}, \mathrm{m}, \mathrm{CH}), 8.59(1 \mathrm{H}, \mathrm{s}, \mathrm{NCH}) .{ }^{13} \mathrm{C}-\mathrm{NMR}$ $\left(75 \mathrm{MHz}, \mathrm{CDCl}_{3}\right): 55.5,104.2,114.6,125.3$, $127.9,128.6,129.3,129.4,129.9,142.7,150.9$, 161.7, 168.0. DEPT 135: 55.5, 104.2, 114.6, 128.6, 129.3, 129.4, 129.9, 150.9. Anal. Cacld. For $\left(\mathrm{C}_{17} \mathrm{H}_{16} \mathrm{~N}_{4} \mathrm{OS}(\%)\right.$ : C, 62.96; H, 4.94; N, 17.28; S : 9.88. Found: C, 62.39; H, 5.11; N, $17.03, \mathrm{~S}, 9.78$.

\section{2-(2-benzylidenehydrazono)-4-(4-(dimethyl-} amino)phenyl)thiazol-3(2H)-amine (10d)

Yellow. Yield: $50 \%$, mp 202-203 ${ }^{\circ} \mathrm{C}$. $\operatorname{IR}\left(\mathrm{cm}^{-1}\right):$ 1596-160 (C=N, aromatic), 3205-3285 $\left(\mathrm{NH}_{2}\right) .{ }^{1} \mathrm{H}-\mathrm{NMR}\left(400 \mathrm{MHz}, \mathrm{CDCl}_{3}\right): 3.01(6 \mathrm{H}$, s, $\left.\mathrm{CH}_{3}\right), 6.79-6.81(2 \mathrm{H}, \mathrm{d}, J=8.9 \mathrm{~Hz}, \mathrm{CH}), 7.15$ $(1 \mathrm{H}, \mathrm{s}, \mathrm{SCH}), 7.54-7.59(5 \mathrm{H}, \mathrm{m}, \mathrm{CH}), 7.72-7.74$ ( $2 \mathrm{H}, \mathrm{m}, \mathrm{CH}), 8.47(1 \mathrm{H}, \mathrm{s}, \mathrm{NCH}) .{ }^{13} \mathrm{C}-\mathrm{NMR}(75$ $\left.\mathrm{MHz}, \mathrm{CDCl}_{3}\right): 39.8,104.2,111.9,119.9,127.9$, $128.6,129.1,129.4,129.8,142.6,151.6,152.1$, 167.2. DEPT 135: 39.8, 104.2, 111.9, 128.6, 129.1, 129.4, 129.8, 151.6. Anal. Calc. For $\left(\mathrm{C}_{18} \mathrm{H}_{20} \mathrm{~N}_{5} \mathrm{~S}\right)(\%): \mathrm{C}, 64.09 ; \mathrm{H}, 5.63 ; \mathrm{N}, 20.77$; S, 9.5. Found: C,63.72; H, 5.83; N, 20.48; S, 9.18 .

2-(2-benzylidenehydrazono)-4-(4-hydroxyphenyl)thiazol-3(2H)-amine (10e)

Yellow to light brown. Yield: $52 \%, \mathrm{mp}$ 164-165 ${ }^{\circ} \mathrm{C} . \quad \operatorname{IR}\left(\mathrm{cm}^{-1}\right) \quad$ : 1570-1607 $(\mathrm{C}=\mathrm{N}$, aromatic), 3400-3500 ( $\left(\mathrm{NH}_{2}\right) .{ }^{1} \mathrm{H}-\mathrm{NMR} \quad(400$ $\mathrm{MHz}, \mathrm{CDCl}_{3}$ ): 3.70 (broad, $\left.1 \mathrm{H}, \mathrm{OH}\right), 6.88-6.90$ $(2 \mathrm{H}, \mathrm{d}, J=8.6 \mathrm{~Hz}, \mathrm{CH}), 7.13(1 \mathrm{H}, \mathrm{s}, \mathrm{SCH}), 7.54-$ $7.56(3 \mathrm{H}, \mathrm{m}, \mathrm{CH}), 7.61-7.63(2 \mathrm{H}, \mathrm{d}, J=8.7 \mathrm{~Hz}$, $\mathrm{CH}), 7.70-7.73(2 \mathrm{H}, \mathrm{m}, \mathrm{CH}), 8.49(1 \mathrm{H}, \mathrm{s}, \mathrm{NCH})$. ${ }^{13} \mathrm{C}$-NMR $\left(75 \mathrm{MHz}, \mathrm{CDCl}_{3}\right): 104.5,116.0$, 123.6, 127.8, 128.6, 129.4, 129.6, 129.9, 142.7, 151.3, 160.5, 167.7. DEPT 135: 104.5, 116.0, 128.6, 129.4, 129.6, 129.9, 151.3. Anal. Cacld. For $\left(\mathrm{C}_{16} \mathrm{H}_{14} \mathrm{~N}_{4} \mathrm{OS}\right)$, Calc. (\%): C, 61.94; $\mathrm{H}, 4.52$;
N, 18.06; S, 10.33. Found: C, 61.67; H, 4.48; N, $17.77 ; \mathrm{S}, 10.00$.

\section{2-(2-(4-bromobenzylidene)hydrazono)-4-(4-}

methoxyphenyl)thiazol-3(2H)-amine (10f)

Yellow to light brown. Yield: $87 \%, \mathrm{mp}$ 196-197 ${ }^{\circ} \mathrm{C} . \quad \operatorname{IR}\left(\mathrm{cm}^{-1}\right) \quad: \quad 1580-1607 \quad(\mathrm{C}=\mathrm{N}$, aromatic), 3200-3200 $\left(\mathrm{NH}_{2}\right) .{ }^{1} \mathrm{H}-\mathrm{NMR} \quad(400$ $\left.\mathrm{MHz}, \mathrm{CDCl}_{3}\right): 3.83\left(3 \mathrm{H}, \mathrm{s}, \mathrm{OCH}_{3}\right), 7.06-7.09$ $(2 \mathrm{H}, \mathrm{d}, J=8.8 \mathrm{~Hz}, \mathrm{CH}), 7.19(1 \mathrm{H}, \mathrm{s}, \mathrm{SCH}), 7.66-$ $7.68(2 \mathrm{H}, \mathrm{d}, J=8.6 \mathrm{~Hz}, \mathrm{CH}), 7.71-7.77(4 \mathrm{H}, \mathrm{m}$, $\mathrm{CH}), 8.52(1 \mathrm{H}, \mathrm{s}, \mathrm{NCH}) .{ }^{13} \mathrm{C}-\mathrm{NMR}(75 \mathrm{MHz}$, $\left.\mathrm{CDCl}_{3}\right)$ : 55.5, 105.1, 114.6, 123.6, 125.2, 127.0, $129.3,131.5,131.5,151.6,150.9,161.7,167.9$. DEPT 135: 55.5, 105.1, 114.6, 129.3, 131.5, 131.5, 150.9. Anal. Cacld. For $\mathrm{C}_{17} \mathrm{H}_{15} \mathrm{BrN}_{4} \mathrm{OS}$ (\%): C, 50.62; H, 3.72; N, 13.90; S, 7.94. Found: C, 50.31; H, 3.62; N,13.69; S, 7.76.

\section{2-(2-(4-bromobenzylidene)hydrazono)-4-(4-(di-} methylamino)phenyl)thiazol-3(2H)-amine $(\mathbf{1 0 g})$

Yellow. Yield: $89 \%$, mp 194-196 ${ }^{\circ} \mathrm{C} .{ }^{1} \mathrm{H}-$ NMR $\left(400 \mathrm{MHz}, \mathrm{CDCl}_{3}\right): 3.01\left(6 \mathrm{H}, \mathrm{s}, \mathrm{CH}_{3}\right)$, 6.78-6.81 $(2 \mathrm{H}, \mathrm{d}, J=8.9 \mathrm{~Hz}, \mathrm{CH}), 7.16(1 \mathrm{H}, \mathrm{s}$, $\mathrm{SCH}), 7.57-7.59(2 \mathrm{H}, \mathrm{d}, J=8.9 \mathrm{~Hz}, \mathrm{CH}), 7.66-$ $7.69(2 \mathrm{H}, \mathrm{d}, J=8.5 \mathrm{~Hz}, \mathrm{CH}), 7.74-7.76(2 \mathrm{H}, \mathrm{d}$, $J=8.5 \mathrm{~Hz}, \mathrm{CH}), 8.45(1 \mathrm{H}, \mathrm{s}, \mathrm{NCH}) .{ }^{13} \mathrm{C}-\mathrm{NMR}$ $\left(75 \mathrm{MHz}, \mathrm{CDCl}_{3}\right): 39.7,39.9,104.2,111.8$, $119.8,123.4,127.3,129.1,131.4,131.5,141.4$, 151.8, 152.1, 167.3. DEPT 135: 39.7, 39.9, 104.2, 111.8, 129.1, 131.4, 131.5, 151.8. Anal. Cacld. For $\mathrm{C}_{18} \mathrm{H}_{19} \mathrm{BrN}_{5} \mathrm{~S}(\%)$ : C, 51.82; H, 4.32; N, 16.79; S, 7.67. Found: C, 51.49; H, 4.39; N, $16.52 ; \mathrm{S}, 7.56$.

\section{2-(2-(4-bromobenzylidene)hydrazono)-4-(4-} hydroxyphenyl)thiazol-3(2H)-amine (10h)

Off-white. Yield: 92\%, mp 195-197 ${ }^{\circ} \mathrm{C}$. $\operatorname{IR}\left(\mathrm{cm}^{-1}\right): 1540-1610(\mathrm{C}=\mathrm{N}$, aromatic $)$, 33503500 $\left(\mathrm{NH}_{2}\right)$. ${ }^{1} \mathrm{H}-\mathrm{NMR}\left(400 \mathrm{MHz}, \mathrm{CDCl}_{3}\right): 3.90$ (broad, $1 \mathrm{H}, \mathrm{OH}), 6.88-6.90(2 \mathrm{H}, \mathrm{d}, J=8.7 \mathrm{~Hz}$, $\mathrm{CH}), 7.19(1 \mathrm{H}, \mathrm{s}, \mathrm{SCH}), 7.60-7.63(2 \mathrm{H}, \mathrm{d}, J=8.7$ $\mathrm{Hz}, \mathrm{CH}), 7.66-7.68(2 \mathrm{H}, \mathrm{d}, J=8.6 \mathrm{~Hz}, \mathrm{CH}), 7.75-$ $7.77(2 \mathrm{H}, \mathrm{d}, J=8.6 \mathrm{~Hz}, \mathrm{CH}), 8.49(1 \mathrm{H}, \mathrm{s}, \mathrm{NCH})$. ${ }^{13} \mathrm{C}-\mathrm{NMR} \quad\left(75 \mathrm{MHz}, \mathrm{CDCl}_{3}\right): 104.8,116.0$, 123.5, 123.6, 127.1, 129.5, 131.4, 131.5, 141.5, 151.4, 160.5, 167.7. DEPT 135: 104.8, 116.0, 129.5, 131.4, 131.5, 151.4. Anal. Cacld. For $\mathrm{C}_{16} \mathrm{H}_{13} \mathrm{BrN}_{4} \mathrm{OS}(\%)$ : C, 49.36; H, 3.34; N, 14.40; S, 8.23. Found: C, $49.11 ; \mathrm{H}, 3.31 ; \mathrm{N}, 14.14$, S, 8.06 . 
General procedure for the preparation of 5(4-bromophenyl)-2-(2-(heteroaryl-2-ylmethylene)hydrazinyl)-6H-1,3,4-thiadiazines 11(a-e)

Mono-thiocarbohydrazone 8(i,j) (5 mmol) was added to a solution of phenacyl bromide, 4bromo phenacyl bromide or 4-chloro phenacyl chloride $(5 \mathrm{mmol})$ in ethanol $(20 \mathrm{ml})$. The reaction mixture was stirred at room temperature for two hours. The product was collected by filtration, washed with ethanol and ether and finally dried.

1-(furan-2-ylmethylene)-2-(5-phenyl-6H-1,3,4thiadiazin-2-yl)hydrazine (11a)

Yellow. Yield: 24\%, mp 187-189 ${ }^{\circ} \mathrm{C}$. $\operatorname{IR}\left(\mathrm{cm}^{-1}\right): 1557-1580(\mathrm{C}=\mathrm{N}$, aromatic $) .{ }^{1} \mathrm{H}-\mathrm{NMR}$ $\left(400 \mathrm{MHz}, \mathrm{CDCl}_{3}\right): 3.96\left(2 \mathrm{H}, \mathrm{s}, \mathrm{CH}_{2}\right), 6.63-6.64$ $(1 \mathrm{H}, \mathrm{m}, \mathrm{CH}), 6.86-6.87(1 \mathrm{H}, \mathrm{d}, J=3.3 \mathrm{~Hz}, \mathrm{CH})$, 7.44-7.49 (3H, m, CH), 7.81-7.83 (3H, m, CH), $8.17(1 \mathrm{H}, \mathrm{s}, \mathrm{NCH}), 11.52(1 \mathrm{H}, \mathrm{s}, \mathrm{NH}) .{ }^{13} \mathrm{C}-\mathrm{NMR}$ $\left(75 \mathrm{MHz}, \mathrm{CDCl}_{3}\right): 21.7,112.2,113.8,125.8$, 128.7, 129.6, 134.8, 142.3, 145.1, 146.7, 150.0, 161.7. DEPT 135: 104.8, 116.0, 129.5, 131.4, 131.5, 151.4. Anal. Cacld. For $\mathrm{C}_{14} \mathrm{H}_{12} \mathrm{~N}_{4} \mathrm{OS}(\%)$ : C, 59.16; H, 4.22; N, 19.71; S, 11.28. Found: C, 58.81; H, 4.18; N, 19.33; S, 10.96.

1-(5-(4-bromophenyl)-6H-1,3,4-thiadiazin-2-yl)2-(furan-2-ylmethylene)hydrazine (11b)

Light green. Yield: $80 \%$, mp 228-229 ${ }^{\circ} \mathrm{C}$. $\operatorname{IR}\left(\mathrm{cm}^{-1}\right)$ : 1557-1580 (C=N, aromatic). ${ }^{1} \mathrm{H}-$ NMR $\left(400 \mathrm{MHz}, \mathrm{CDCl}_{3}\right): 3.95\left(2 \mathrm{H}, \mathrm{s}, \mathrm{CH}_{2}\right)$, 6.62-6.64 $(1 \mathrm{H}, \mathrm{m}, \mathrm{CH}), 6.87-6.88(1 \mathrm{H}, \mathrm{d}, \mathrm{J}=3.3$ $\mathrm{Hz}, \mathrm{CH}), 7.65-7.67(2 \mathrm{H}, \mathrm{d}, J=8.6 \mathrm{~Hz}, \mathrm{CH}), 7.75-$ $7.77(2 \mathrm{H}, \mathrm{d}, J=8.6 \mathrm{~Hz}, \mathrm{CH}), 7.83-7.84(1 \mathrm{H}, \mathrm{d}$, $J=1.3 \mathrm{~Hz}, \mathrm{CH}), 8.17(1 \mathrm{H}, \mathrm{s}, \mathrm{NCH}), 11.59(1 \mathrm{H}, \mathrm{s}$, $\mathrm{NH}) .{ }^{13} \mathrm{C}-\mathrm{NMR}\left(75 \mathrm{MHz}, \mathrm{CDCl}_{3}\right): 21.9,112.7$, $114.4,123.5,128.3,132.1,134.6,143.0,145.6$, 145.9, 150.5, 161.7. DEPT 135: 21.4, 112.2, 113.9, 127.8, 131.6, 142.5, 145.1. Anal. Cacld. For $\mathrm{C}_{14} \mathrm{H}_{11} \mathrm{BrN}_{4} \mathrm{OS}$ : (\%): C, 46.30; H, 3.03; N, 15.43; S, 8.83. Found: C, 46.01; H, 2.99; N, $15.16 ; \mathrm{S}, 8.51$.

1-(5-phenyl-6H-1,3,4-thiadiazin-2-yl)-2-

(thiophen-2-ylmethylene)hydrazine (11c)

Yellow. Yield: 63\%, mp 216-217 ${ }^{\circ} \mathrm{C}$. $\operatorname{IR}\left(\mathrm{cm}^{-1}\right): 1557-1590 \quad(\mathrm{C}=\mathrm{N}$, aromatic $) .{ }^{1} \mathrm{H}-$ NMR $\left(400 \mathrm{MHz}, \mathrm{CDCl}_{3}\right): 3.96\left(2 \mathrm{H}, \mathrm{s}, \mathrm{CH}_{2}\right)$, 7.12-7.14 (1H, m, CH), 7.42-7.49 $(4 \mathrm{H}, \mathrm{m}, \mathrm{CH})$, 7.61-7.62 (1H, d, $J=4.9 \mathrm{~Hz}, \mathrm{CH}), 7.80-7.83(2 \mathrm{H}$, $\mathrm{m}, \mathrm{CH}), 8.45(1 \mathrm{H}, \mathrm{s}, \mathrm{NCH}), 11.52(1 \mathrm{H}, \mathrm{s}, \mathrm{NH})$. ${ }^{13} \mathrm{C}-\mathrm{NMR}\left(75 \mathrm{MHz}, \mathrm{CDCl}_{3}\right): 21.7,125.8,127.8$, 128.6, 128.7, 129.5, 130.4, 134.9, 139.9, 146.6, 147.3, 161.3. DEPT 135: 21.7, 125.8, 127.8,
128.6, 128.7, 129.5, 130.4, 147.3. Anal. Cacld. For $\mathrm{C}_{14} \mathrm{H}_{12} \mathrm{~N}_{4} \mathrm{~S}_{2}$ : (\%) C, 56.00; H, 4.00; N, 18.66; S, 21.35 Found: C, 55.56; H, 3.91; N, 18.28; S, 21.01.

1-(5-(4-bromophenyl)-6H-1,3,4-thiadiazin-2-yl)2-(thiophen-2-ylmethylene)hydrazine (11d)

Yellow. Yield: $72 \%$, mp 240-243 ${ }^{\circ} \mathrm{C}$. $\operatorname{IR}\left(\mathrm{cm}^{-1}\right): 1555-1593(\mathrm{C}=\mathrm{N}$, aromatic $) .{ }^{1} \mathrm{H}-\mathrm{NMR}$ $\left(400 \mathrm{MHz}, \mathrm{CDCl}_{3}\right): 3.95\left(2 \mathrm{H}, \mathrm{s}, \mathrm{CH}_{2}\right), 7.12-7.15$ $(1 \mathrm{H}, \mathrm{m}, \mathrm{CH}), 7.42-7.43(1 \mathrm{H}, \mathrm{m}, \mathrm{CH}), 7.62-7.63$ $(1 \mathrm{H}, \mathrm{m}, \mathrm{CH}), 7.66-7.68(2 \mathrm{H}, \mathrm{m}, \mathrm{CH}), 7.75-7.77$ $(2 \mathrm{H}, \mathrm{m}, \mathrm{CH}), 8.49(1 \mathrm{H}, \mathrm{s}, \mathrm{NCH}), 11.59(1 \mathrm{H}, \mathrm{s}$, $\mathrm{NH}) .{ }^{13} \mathrm{C}-\mathrm{NMR}\left(75 \mathrm{MHz}, \mathrm{CDCl}_{3}\right): 21.4,122.9$, $127.8,127.9,128.7,130.5,131.6,134.1,139.9$, 145.3, 147.5, 160.8. DEPT 135: 21.4, 127.8, 127.9, 128.7, 130.5, 131.6, 147.5. Anal. Cacld. For $\mathrm{C}_{14} \mathrm{H}_{11} \mathrm{BrN}_{4} \mathrm{~S}_{2}(\%)$ : C, 44.32; $\mathrm{H}, 2.90 ; \mathrm{N}$, 14.77; S, 16.88. Found: C, 43.98; H, 2.81; N, 14.43; S, 16.57.

1-(5-(4-chlorophenyl)-6H-1,3,4-thiadiazin-2-yl)2-(thiophen-2-ylmethylene)hydrazine (11e)

Yellow. Yield: 32\%, mp 224-226 ${ }^{\circ} \mathrm{C}$. $\operatorname{IR}\left(\mathrm{cm}^{-1}\right)$ : 1557-1590 (C=N, aromatic). ${ }^{1} \mathrm{H}-$ NMR $\left(400 \mathrm{MHz}, \mathrm{CDCl}_{3}\right): 3.96\left(2 \mathrm{H}, \mathrm{s}, \mathrm{CH}_{2}\right)$, 7.12-7.14 $(1 \mathrm{H}, \mathrm{m}, \mathrm{CH}), 7.42-7.44(1 \mathrm{H}, \mathrm{d}, J=8.5$ $\mathrm{Hz}, \mathrm{CH}), 7.44-7.49(2 \mathrm{H}, \mathrm{d}, J=4 \mathrm{~Hz}, \mathrm{CH}), 7.61-$ $7.62(1 \mathrm{H}, \mathrm{d}, J=5 \mathrm{~Hz}, \mathrm{CH}), 7.80-7.83(2 \mathrm{H}, \mathrm{m}$, $\mathrm{CH}), 8.49(1 \mathrm{H}, \mathrm{s}, \mathrm{NCH}), 11.51(1 \mathrm{H}, \mathrm{s}, \mathrm{NH}) .{ }^{13} \mathrm{C}-$ NMR $\left(75 \mathrm{MHz}, \mathrm{CDCl}_{3}\right)$ : 21.7, 125.8, 127.8, $128.6,128.7,129.5,130.4,134.9,139.9,146.6$, 147.3, 161.3. DEPT 135: 21.7, 125.8, 127.7, 128.6, 128.7, 129.5, 130.4, 147.3. Anal. Cacld. For $\mathrm{C}_{14} \mathrm{H}_{11} \mathrm{ClN}_{4} \mathrm{~S}_{2}(\%)$ : C, 50.23; $\mathrm{H}, 3.29 ; \mathrm{N}$, 16.74; S, 19.16. Found: C, 49.91; H, 3.21; N, $16.43 ; \mathrm{S}, 18.86$.

\section{Pharmacophore Modeling Study}

Four derivatives were screened against 95 pharmacophore models developed by Dr. Mutasem Taha (Faculty of Pharmacy, the University of Jordan) for targets involved in diverse disease conditions. Pharmacophore models predict the important structural properties of the compounds, including their hydrophobic and aromatic features. The aromatic ligand may interact with the aromatic amino acid residues of the target. The pharmacophore model can depict the assessment of 3D features encoding bioactivity of a series of active molecules having common binding mode to the biological target ${ }^{[32-34]}$. Therefore, 3D pharmacophore is based upon the assumption that all the structurally diverse molecules can 
bind in a common binding mode to the biological target. These 3D properties include six pharmacophoric features; namely, hydrogen bond donor, hydrogen bond acceptor, hydrophobicity of the moiety, aromatic rings, positive ionization properties (cation) and negative ionization properties (anion) ${ }^{[35-38]}$.

\section{Results and Discussion}

Synthesis of the substituted 1, 3-thiazoles 10a-h was carried out through the reaction of the mono-thiocarbohydrazones 8a-h with one equivalent of $\alpha$-bromoacetophenone (9) in ethanol. The reaction affords moderate to good yields of the corresponding substituted 1,3thiazoles 10a-h, Scheme 2. Compounds 10a-h were isolated as fine-colored crystals, washed with ether and recrystallized from ethanol.

The reaction is expected to proceed via the mechanism shown in Scheme 3. The reaction initially involves a nucleophilic attack of the thiol moiety on the mono-thiocarbohydrazones tautomer on the $\alpha$-carbon of the carbonyl compound, followed by ring closure. Colors, melting points and yields of compounds 10a-h are included in the experimental part.<smiles>[Y]c1ccc(-c2cs/c(=N\N=C\[Al]CCBr)n2N)cc1</smiles>

\begin{tabular}{ccc}
\hline Comp. 10 & Ar & Y \\
\hline $\mathbf{a}$ & $\mathrm{C}_{6} \mathrm{H}_{5}$ & $\mathrm{H}$ \\
b & $4-\mathrm{CH}_{3} \mathrm{C}_{6} \mathrm{H}_{4}$ & $\mathrm{H}$ \\
$\mathbf{c}$ & $4-\mathrm{CH}_{3} \mathrm{OC}_{6} \mathrm{H}_{4}$ & $\mathrm{H}$ \\
$\mathbf{d}$ & $4-\left(\mathrm{CH}_{3}\right)_{2} \mathrm{NC}_{6} \mathrm{H}_{4}$ & $\mathrm{H}$ \\
$\mathbf{e}$ & $4-\mathrm{HOC}_{6} \mathrm{H}_{4}$ & $\mathrm{H}$ \\
$\mathbf{f}$ & $4-\mathrm{CH}_{3} \mathrm{OC}_{6} \mathrm{H}_{4}$ & $\mathrm{Br}$ \\
$\mathbf{g}$ & $4-\left(\mathrm{CH}_{3}\right)_{2} \mathrm{NC}_{6} \mathrm{H}_{4}$ & $\mathrm{Br}$ \\
$\mathbf{h}$ & $4-\mathrm{HOC}_{6} \mathrm{H}_{4}$ & $\mathrm{Br}$ \\
\hline
\end{tabular}

Scheme 2. Reactions of mono-thiocarbohydrazones with $\alpha$-bromoketones: Synthesis of substituted 1, 3-thiazoles.

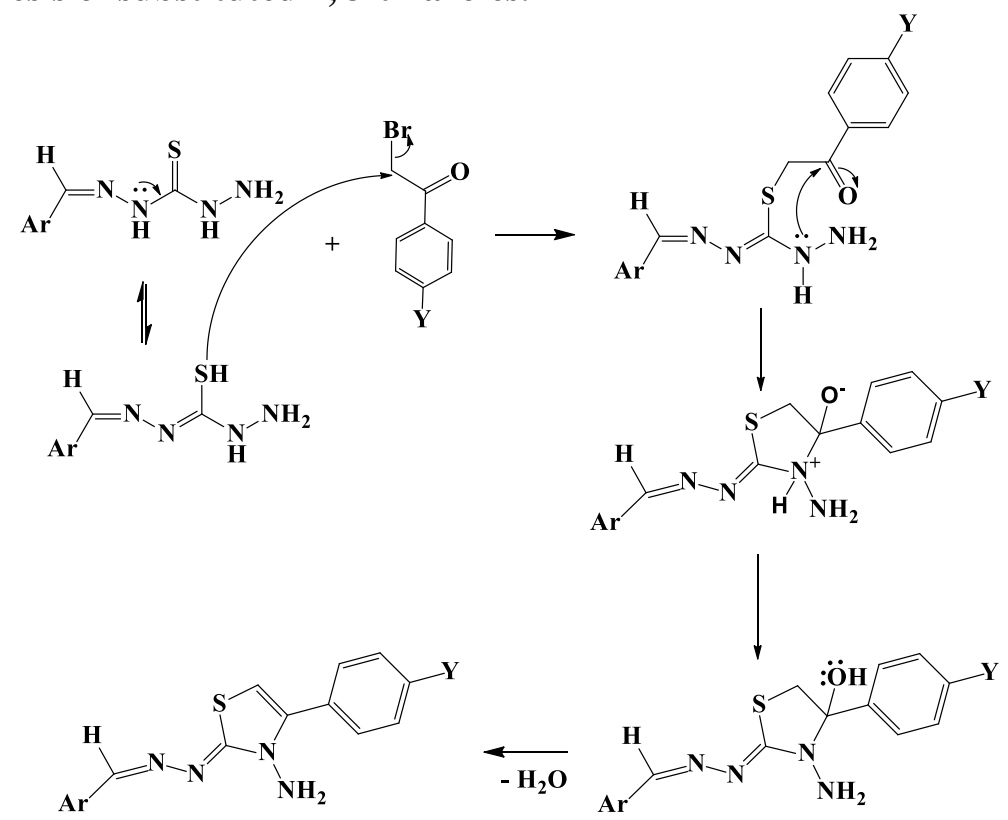

Scheme 3. Suggested mechanism of the reaction of monothiocarbohydrazones with $\alpha$-bromoketones. 
Compounds 10a-h are fully characterized using spectroscopic techniques and elemental analysis. Thus, the ${ }^{1} \mathrm{H}-\mathrm{NMR}$ spectra of compounds 10a-h revealed a one proton singlet at $\delta=7.13-7.21 \mathrm{ppm}$ assigned to the thiazole ring proton. In ${ }^{13} \mathrm{C}-\mathrm{NMR}$, the quaternary $\mathrm{C}-2$ carbon in the thiazole ring resonated in the range $\delta=167.3-168.5 \mathrm{ppm}$, while C-4 resonated in the range $\delta=142.7-161.6 \mathrm{ppm}$ and $\mathrm{C}-5$ resonated in the range $\delta=103.7-105.1 \mathrm{ppm}$.<smiles>[Y]C=NN=C1SCC(c2ccc(I)cc2)=C1I</smiles>

Figure 1. Numbering of $\mathrm{C}$ atoms in 10a-h.

The hydrazino carbon resonated at $\delta=$ 150.9-151.7 ppm. Aromatic carbons and other carbons of related substituents appeared at the expected chemical shifts. The assignment of $\mathrm{SCH}(\mathrm{C}-5)$ and the hydrazino carbon $(\mathrm{N}-\mathrm{N}=\mathrm{C}-\mathrm{H})$ signals were confirmed by determining the HMQC-NMR spectrum. ${ }^{1} \mathrm{H}-\mathrm{NMR},{ }^{13} \mathrm{C}-\mathrm{NMR}$ and DEPT 135 of compounds $\mathbf{1 0 a}-\mathbf{h}$ are given in the experimental part.

The infrared spectra show characteristic bands around 1540 and $1611 \mathrm{~cm}^{-1}$ due to the stretching of the $\mathrm{C}=\mathrm{N}$ (imine) and aromatic bonds. The $\mathrm{NH}_{2}$ group appears around 3200$3500 \mathrm{~cm}^{-1}$ as two weak absorptions.

In contrast to the above-mentioned reactions of mono-thiocarbohydrazones 8a-h, furylthiocarbohydrazone (8i) and thienyl-thiocarbohydrazone $(\mathbf{8 j})$ were found to react differently. Thus, compound $\mathbf{8 i}$ readily reacted with phenacyl bromide under the same conditions that are previously mentioned in the former reactions, to give exclusively the substituted six-member ring 1,3,4-thiadiazine derivative 11a. Similar results were observed in the reactions of $\mathbf{8 i}$ and 8j with p-bromophenacyl bromide and pchlorophenacyl chloride, Scheme 4.

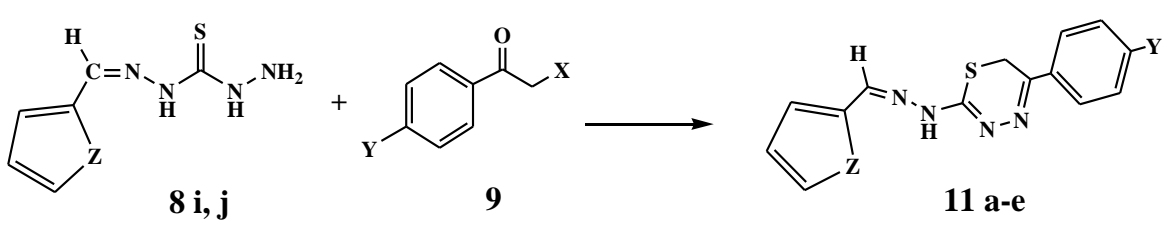

\begin{tabular}{cll}
\hline Comp. 11 & Z & Y \\
\hline $\mathbf{a}$ & $\mathrm{O}$ & $\mathrm{H}$ \\
$\mathbf{b}$ & $\mathrm{O}$ & $\mathrm{Br}$ \\
$\mathbf{c}$ & $\mathrm{S}$ & $\mathrm{H}$ \\
$\mathbf{d}$ & $\mathrm{S}$ & $\mathrm{Br}$ \\
$\mathbf{e}$ & $\mathrm{S}$ & $\mathrm{Cl}$ \\
\hline
\end{tabular}

Scheme 4. Reaction of furyl- and thienyl-thiocarbohydrazone with phenacyl bromide and chloride $(\mathrm{X}=\mathrm{Br}, \mathrm{Cl})$.

The reason behind the different reaction behavior of furyl- and thienyl-thiocarbohydrazone with phenacyl halides, compared to that of $\mathbf{8 a - h}$, is still not determined. However, it is believed that the reaction proceeds via the mechanism shown in Scheme 5. Further investigation is needed to demonstrate the difference in the orientation of ring closure in the case of hetero-aromatic substituted thiocarbo- hydrazones, in comparison with the reactions of compounds 8a-h. It is worth mentioning that Kaplanciki and coworkers reported that N-(1furyl ethylidene) thiocarbo-hydrazide and $\mathrm{N}-(1-$ thienylethylidene) thiocarbo-hydrazide $(\mathbf{1 2 a}, \mathbf{b})$ readily reacted with phenacyl bromide $(\mathbf{8})$ in dimethylformamid (DMF) to afford substituted 1,2,4-triazine-3-thione derivatives (13a, ,b), as shown in the following equation ${ }^{[30]}$ :

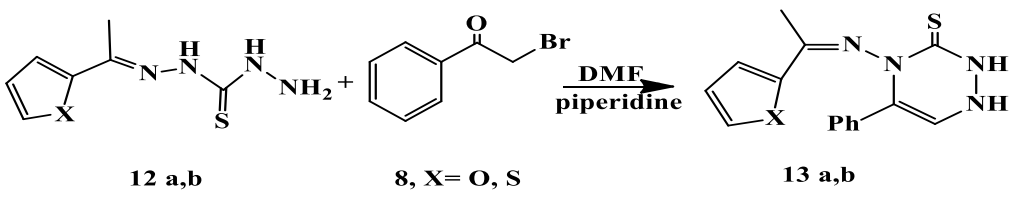




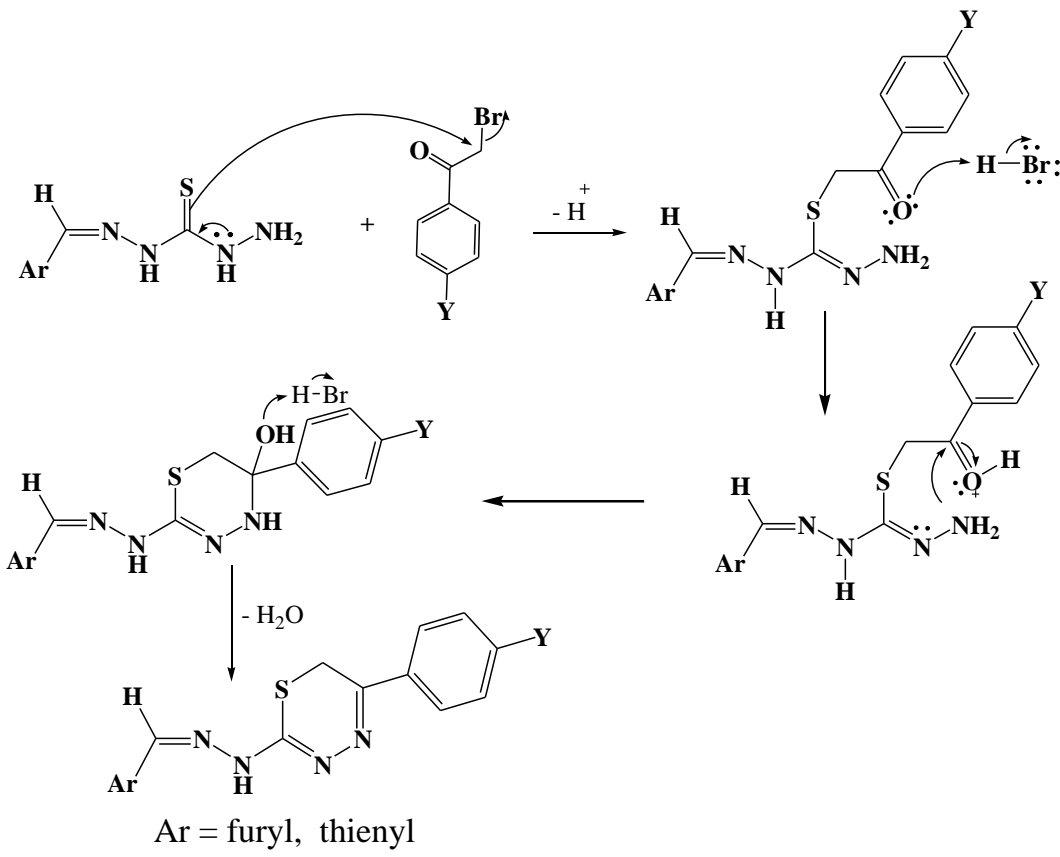

Scheme 5. Suggested mechanism for the reaction of furyl- and thienylthiocarbohydrazone with phenacyl bromide and chloride $(\mathrm{X}=\mathrm{Br}, \mathrm{Cl})$.

Colors, melting points and yields of compounds 11a-e are included in the experimental part. The structures of products 11a-e are fully determined using spectroscopic methods. The ${ }^{1} \mathrm{H}-\mathrm{NMR}$ spectra of compounds 11a-e revealed a singlet in the range $\delta=3.95$ $3.96 \mathrm{ppm}$ assigned for the methylene protons of the six-member ring. No ${ }^{1} \mathrm{H}-\mathrm{NMR}$ signals of the thiazole $\mathrm{CH}$ ring proton were observed for the crude product. Thus, thiadiazine derivatives are the exclusive products. Another two singlets were observed, one appeared at $\delta=8.17-8.49$ ppm assigned to the $\mathrm{CH}$ of hydrazino group and the other at $\delta=11.51-11.59 \mathrm{ppm}$ assigned to $\mathrm{NH}$. The other aromatic and the substituent protons resonated at the expected chemical shifts.

In ${ }^{13} \mathrm{C}$-NMR, C-6 in the thiadiazine ring $\left(\mathrm{CH}_{2}\right)$ resonated in the range $\delta=21.4-21.9 \mathrm{ppm}$, while the quaternary $\mathrm{C}-2$ resonated in the range $\delta$ $=160.8-161.7 \mathrm{ppm}$. The hydrazino carbon $(\mathrm{C}=\mathrm{N}-\mathrm{N})$ resonated in the range $\delta=112.2-127.8$ ppm. ${ }^{1} \mathrm{H}-\mathrm{NMR},{ }^{13} \mathrm{C}-\mathrm{NMR}$ and DEPT-135 of compounds 11a-e are given in the experimental section.

The infrared spectra of compounds 11a-e show characteristic bands around 1555-1597 $\mathrm{cm}^{-1}$ corresponding to tretch vibrations of the $\mathrm{C}=\mathrm{N}$ (imine) and aromatic bonds.<smiles></smiles>

Figure 2. Numbering of $\mathrm{C}$ atoms in 11a-e.

\section{Pharmacophore Modeling}

The results of the pharmacophore modeling study are presented in Table 1 , which shows a list of the tested compounds and the percentage probabilities of their potential bioactivities against corresponding targets. The results indicate that the compounds have a good potential as biologically active compounds. Out of the 95 screened models, our compounds fitted 4 targets: NMT involved in fungal infections, FLT3 involved in blood cancer (leukemia), BACE involved in Alzheimer's disease and AChE involved in Parkinson's disease. The biological properties of the compounds will be investigated on the above parameters as well as on some enzymes related to some other diseases. 
Table 1: The results of the pharmacophore studies on some thiazole and thiadiazine derivatives*.

\begin{tabular}{|c|c|c|c|c|c|c|c|}
\hline & Structure & $\begin{array}{c}\text { RHO- } \\
\text { KINASE }\end{array}$ & PPAR- $\gamma$ & NMT & FLT3 & BACE & $\mathrm{AChE}$ \\
\hline B12 & & 0 & 0 & 50 & 0 & 59 & 0 \\
\hline B6 & & 46 & 3 & 0 & 85 & 34 & 53 \\
\hline SB2 & & 0 & 7 & 0 & 0 & 18 & 0 \\
\hline FB4 & & 1 & 44 & 71 & 0 & 72 & 0 \\
\hline
\end{tabular}

*RHO-KINASE: Involved in hypertension

PPAR- $\gamma$ : Anti-diabetes target

NMT : Anti-fungal target N-myristoyl transferase

FLT3: Anti-cancer kinase target

BACE: Beta-secretase, anti-Alzheimer disease target

AChE: Acetylcholine esterase, target for treating Parkinsonism.

\section{Conclusion}

The results of this study reveal the discrepancy in the reactions of mono aryl-thiocarbohydrazones and heteroaryl thiocarbohydrazones. The former yielded substituted 1,3thiazoles, whereas the latter afforded substituted 1,3,4-thiadiazines. A plausible mechanism has been suggested for each case. Pharmacophore modeling study revealed that some of these compounds have potential biological activities.
The biological activities of these compounds will be further investigated experimentally

\section{Acknowledgments}

The financial support of this work by the Deanship of Graduate Studies and Scientific Research at Yarmouk University (project number 29/2014) is highly acknowledged. The authors would like to thank Prof. Dr. Mutasem Taha for his help in the pharmacophore studies.

\section{References}

[1] Trost, B. M., Chem Rev., 1978, 78, 363-382.

[2] Lindhorst, K. T.; Kieburg, C.; KrallmannWenzel, U., Glycoconjugate J., 1998, 15, 605-613.

[3] Aly, A. A.; Brown, A. B.; El-Emary, T. I.; Ewas, A. M. M.; Ramadan, M., ARKIVOC, 2009, 150-197.

[4] Invidiata, F. B.; Furno, G.; Busse, I.; Simoni, D., J. Heterocyclic Chem., 1997, 34, 125562.
[5] Kaplacikli, Z.; Turan-Zitouni, G., J. Enzyme Inhibition Med. Chem., 2005, 20, 179-182.

[6] Moustafa, A. H.; Haggam, R. A.; Younes, M. E.; El-Ashry, E. S. H., Nucleosides, Nucleotides and Nucleic Acid, 2005, 24, 1885-1895.

[7] Al-Ashry, E. S. H.; Awad, L. F.; Abdelhamid, H. M., Nucleosides, Nucleotides and Nucleic Acid, 2006, 25, 325-335. 
[8] Abdel-Hady, S., Sulfur Lett., 1990, 11, 117122.

[9] El-Gendy, Z.; Morsy, J. M.; Allimony, H. A.; Abdel-Monem, W. A.; Abdel-Rahman, R. M., Pharmazie, 2001, 56, 376-382.

[10] Kabashima, S., Okawara, T., Yamasaki, T., Furkawa, M., Heterocycles, 1990, 31, 1129-1139.

[11] El-Sayed Ali, T., J. Sulf. Chem., 2009, 30, 611-647.

[12] Shkoor, M.; Al-Abade, A. ; Aleteiwi, I. ; Al-Talib, M.; Tashtoush, H., Synth. Comm., 2017,47, 1471-1477.

[13] a) Zelenin K.; Alekseyev, V., Tetrahedron lett., 1990, 31, 3927-3930; b) Lamon, R., J. Org. Chem., 1969, 34, 756-758.

[14] Tashtoush, H. I.; Abusahyon, F.; Shkoor, M.; Al-Talib, M., J. Sulf. Chem., 2011, 32, 405-412.

[15] a) Sondhi, S. M.; Verma, R. P.; Nidhi, S.; Shukla, R.; Raghubir, R.; Dubey, M. P., Indian Drugs, 1999, 36, 50-54; b) Sayed, A. R., Tetrahedron, 2012, 68, 2784-2789.

[16] Page, D.; Roy, R., Glycoconjugate J., 1997, 14, 345-356.

[17] Page, D.; Roy, R., Bioconjugate Chem., 1997, 8, 714-723.

[18] Fedorova, O. V.; Mordavaski, G. G.; Rusiov, G. L.; Zueva, M. N.; Ovchinnikova, I. G., Khim-Farm Zh., 1996, 30, 6; Chem. Abstr., 1997, 126, 152416y.

[19] a) El-Sayed, T. A., J. Sulf. Chem., 2009, 30, 611-647; b) Kurzer, F.; Wilkinson, M., Chem. Rev., 1970, 70, 111-149.

[20] Suthar, S. K.; Jaisal, V.; Lohan, S.; Bansal, S.; Chaudary, A.; Tiwari, A.; Alex, A. T.; Joesph, A., Eur. J. Med. Chem., 2013, 63, 589-602.

[21] Sharma, S.; Sharma, P. K.; Kumar, N.; Dudhe, R. A., Biomed. Pharmacother., 2011, 65, 244-251.

[22] Patel, D.; Kumari, P.; Patel, N., Eur. J. Med. Chem., 2012, 48, 354-362.
[23] Rawal, R. K.; Tripathi, R.; Katti, S. B.; Pannaecouque, C.; de Clercq, E., Bioorg. Med. Chem., 2007, 15, 1725-1731.

[24] Apotrosoaei, M.; Vasincu, I. M.; Dragan, M.; Buron, F.; Routier, S.; Profire, L., Molecules, 2014, 19, 13824-13847.

[25] Masincu, I. M.; Apotrosoaei, M.; Panzariu, A-T.; Buron, F.; Routier, S.; Profire, L., Molecules, 2014, 19, 15005-15025.

[26] Shingalapur, R. V.; Hosmani, K. M.; Keri, R. S.; Hugar, M. H., Eur. J. Med. Chem., 2010, 45, 1753-1759.

[27] Kishore, A.; Nampurath, G. K.; Mathew, S. P.; Zachariah, R. T.; Potu, B. K.; Rao, M. S.;Valiathan, M.; Chamallamudi, M. R., Chem. Biol. Interact., 2009, 177, 242-246.

[28] Cong, N. T.; Nhan, H. T.; Hung, L. V.; Thang, T. D.; Kuo, P-C., Molecules, 2014, 19, 13824-13847.

[29] Badawy, M. A.; Abdel-Hady, S. A.; Ibrahim, Y. A., Liebigs Ann. Chem., 1990, 393-395.

[30] Kaplancikli, Z. A; Turan-Zitouni, G.; Ozdemir, A.; Altintop, M. D.; Tunali, Y.; Asian J. Chem., 2010, 22, 6701-6707.

[31] Rajendran, G.; Jian, S. R., Indian J. Chem., 1985, 24B, 680-684.

[32] Leach, A. R.; Gillet, V. J.; Lewis, R. A. ; Taylor, R., J. Med. Chem., 2009, 53, 539558.

[33] Shoichet, B. K., Nature, 2004, 432, 862865.

[34] Mason, J.S. ; Good, A. C. ; Martin E. J., Curr. Pharm. Des., 2001, 7, 567-597.

[35] Langer, T.; Krovat, E. M., Curr. Opin. Drug Discov. Dev., 2003, 6, 370-376.

[36] Koes, D. R.; Camacho, C.J., Pharmer: J. Chem. Inf. Model., 2011, 51, 1307-1314.

[37] Nandi, S.; Bagchi, M. C., Chem. Biol. Drug Des., 2011, 78, 587-595.

[38] Batra, A.; Nandi, S.; Bagchi, M. C., Med. Chem. Res., 2015, 24, 2432-2440. 